ARTICLE

Received 23 Aug 2013 | Accepted 30 Oct 2013 | Published 4 Dec $2013 \quad$ DOl: 10.1038/ncomms3846

\title{
The neuropeptide NLP-22 regulates a sleep-like state in Caenorhabditis elegans
}

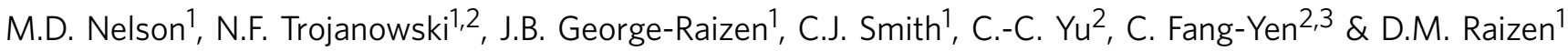

Neuropeptides have central roles in the regulation of homoeostatic behaviours such as sleep and feeding. Caenorhabditis elegans displays sleep-like quiescence of locomotion and feeding during a larval transition stage called lethargus and feeds during active larval and adult stages. Here we show that the neuropeptide NLP-22 is a regulator of Caenorhabditis elegans sleep-like quiescence observed during lethargus. $n \mid p-22$ shows cyclical mRNA expression in synchrony with lethargus; it is regulated by LIN-42, an orthologue of the core circadian protein PERIOD; and it is expressed solely in the two RIA interneurons. $n / p-22$ and the RIA interneurons are required for normal lethargus quiescence, and forced expression of $n / p-22$ during active stages causes anachronistic locomotion and feeding quiescence. Optogenetic stimulation of the RIA interneurons has a movement-promoting effect, demonstrating functional complexity in a single-neuron type. Our work defines a quiescence-regulating role for NLP-22 and expands our knowledge of the neural circuitry controlling Caenorhabditis elegans behavioural quiescence.

\footnotetext{
${ }^{1}$ Department of Neurology, Perelman School of Medicine, University of Pennsylvania, Philadelphia, Pennsylvania 19104, USA. ${ }^{2}$ Department of Bioengineering, University of Pennsylvania, Philadelphia, Pennsylvania 19104, USA. ${ }^{3}$ Department of Physics, Korea University, Anam-dong, Seongbuk-gu, Seoul 136-701, Korea. Correspondence and requests for materials should be addressed to D.M.R. (email: raizen@mail.med.upenn.edu).
} 
$\mathrm{N}$ europeptides are central to mechanisms by which biological clocks temporally align animal behaviour with recurring internal or external conditions. In mammals, the neuropeptide hypocretin/orexin is secreted from lateral hypothalamic neurons to promote wakefulness during the optimal time of day for foraging ${ }^{1}$. Other mammalian neuropeptides shown or proposed to regulate behavioural outputs of circadian clocks include prokineticin ${ }^{2}$, epidermal growth factor $(E G F)^{3}$, neuropeptide $S^{4}$, prolactin-releasing peptide ${ }^{5}$, urotensin II $^{6}$ and neuromedin $S^{7}$. In the fruit fly Drosophila melanogaster, the neuropeptide pigment-dispersing factor (PDF) is released from central nervous system circadian neurons to control sleep/wake behaviour ${ }^{8-10}$, emphasizing a conserved role for neuropeptides in translating clock information to behaviour ${ }^{8}$.

In Caenorhabditis elegans (C. elegans), sleep-like behaviour is observed during lethargus periods, which occur at transitions between each of the four larval stages and between the fourth larval stage and the adult stage ${ }^{11}$. During lethargus, animals, cease locomotion, feeding and foraging, are less responsive to stimuli and display a homoeostatic response to forced movement ${ }^{12,13}$. Signalling pathways involving cAMP-dependent protein kinase (PKA), cyclic GMP-dependent protein kinase (PKG), EGF and PDF, which have roles in the regulation of mammalian and/or Drosophila sleep, also function in the regulation of C. elegans sleep-like quiescence ${ }^{12,14,15}$, suggesting that C. elegans quiescence has fundamental similarities to sleep in other animals. Additional molecular similarity between quiescence during lethargus and mammalian sleep is demonstrated by the larval cycling of expression of the protein LIN-42, which is homologous to the core circadian protein PERIOD ${ }^{16}$. lin-42 is required for proper timing of lethargus ${ }^{17}$, much as period is required for proper timing of circadian sleep ${ }^{18}$, but mechanisms by which lin-42 may affect quiescent behaviour were previously unknown.

Here we identify the gene $n l p-22$ as a lin-42-regulated gene affecting quiescent behaviour. $n l p-22$ encodes a neuropeptide whose function and expression were previously unknown. We show that $n l p-22$ can induce quiescence in normally active animals and is necessary for normal lethargus quiescence. In addition, we present evidence of a surprising functional complexity of neurons expressing NLP-22. Our findings expand our knowledge of the neural circuitry controlling $C$. elegans behavioural quiescence.

\section{Results}

nlp-22 expression cycles in response to a larval clock. The activity of many mammalian neuropeptides with roles in clock regulation is controlled at the level of mRNA abundance. We therefore reasoned that a somnogenic peptide controlled by the larval clock would be upregulated before or during lethargus. We performed a transcriptional profiling experiment (manuscript in preparation) in precisely staged animals to identify genes with elevated expression before or during the fourth lethargus stage. mRNA abundance of the gene $n l p-22$, which encodes a protein predicted to be processed into a neuropeptide ${ }^{19}$, was higher in the L4 stage than in lethargus. Using qRT-PCR, we confirmed that nlp-22 mRNA expression cycles with a constant phase relationship to lethargus (Fig. 1a). nlp-22 mRNA peaked midway through the active larval stages, decreased during each lethargus stage, and was absent in adults.

Given the cyclical nature of $n l p-22$ expression, we hypothesized that NLP-22 signalling is regulated by the larval clock. The C. elegans orthologue of the core circadian regulator PERIOD is LIN-42, which is required for the synchronization of lethargus quiescence and moulting ${ }^{16,17}$. Like PERIOD, which shows cyclical expression with a circadian periodicity in insects and mammals ${ }^{20}$, LIN-42 shows cyclical expression with larval periodicity in C. elegans ${ }^{16,21}$. LIN-42 expression has a similar phase relationship to lethargus as NLP-22. We found that LIN-42A anachronistic overexpression induced an increase in $n l p-22$ mRNA (Fig. 1b), suggesting that $n l p-22$ transcription is downstream of a larval lin-42-based clock.

nlp-22 is expressed in the pair of RIA interneurons. To determine where $n l p-22$ is expressed, we generated a transcriptional reporter by placing green fluorescent protein (GFP) under the control of $n l p-22$ regulatory DNA. We observed expression only in one pair of head neurons, which we identified as the RIA interneurons, based on process morphology and in comparison with the expression of the previously characterized gene $g l r-3$
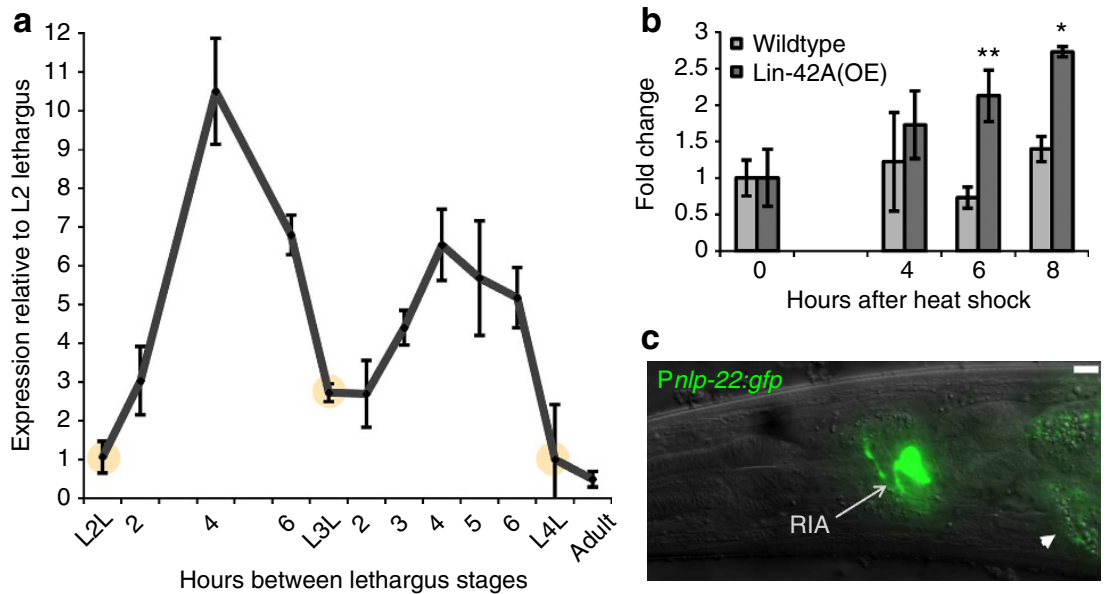

Figure $1|\mathbf{n}| \mathbf{p}-\mathbf{2 2}$ is expressed in a dynamic manner in the RIA interneurons. (a) mRNA expression during larval development. Expression levels cycle in synchrony with each lethargus stage. Yellow circles depict each lethargus stage, as defined by cessation of feeding of the animals (Biological replicates per time point $\geq 3$, technical replicates $=2$ for each biological replicate; error bars represent s.e.m.). (b) nlp-22 expression is increased in response to lin-42 overexpression during the adult stage. ( ${ }^{\star} P<0.05,{ }^{\star \star} P<0.005$, Student's two-tailed $t$-test, biological replicates per condition $\geq 5$, technical replicates $=2$ for each biological replicate; error bars represent s.e.m.). (c) An nlp-22 transcriptional GFP reporter is expressed in the RIA neurons (arrow). Intestinal auto-fluorescence is also observed (arrow head). Scale bar $=5 \mu \mathrm{M}$. 
(Fig. 1c and Supplementary Fig. S1a). Thus, $n l p-22$ expression is cyclical and restricted to the RIA neurons.

NLP-22 is predicted to be secreted ${ }^{19}$. Secreted proteins in C. elegans, when fused to a fluorescent moiety on their C-terminus, can be visualized in cells outside of the cells in which they are expressed ${ }^{22}$. When we fused GFP to the C-terminus of the NLP-22 preproprotein, we observed green fluorescence in several neurons in the head as well as in the ventral nerve cord (Supplementary Fig. S1b), supporting the notion that NLP-22 is a secreted peptide. When we fused GFP to the N-terminus of the NLP-22 preproprotein, expression was exclusively in the RIA neurons, presumably because the GFP protein interfered with the functionality of the signal sequence (Supplementary Fig. S1c). Thus, our data indicate that the NLP-22 peptide is processed and secreted from the RIA interneurons and may be acting by synaptic and/or neuroendocrine mechanisms.

NLP-22 induces behavioural quiescence. On the basis of the observation that $n l p-22$ mRNA expression cycles during larval development in phase with the cycling of sleep-like behaviour, we hypothesized that it regulates lethargus quiescence. To test this hypothesis, we genetically manipulated $n l p-22$ expression and observed the effects on quiescence and active behaviour.

We first studied the effect of temporal and spatial misexpression of $n l p-22$ during the adult stage, when the animals are typically feeding and moving. C. elegans moves by making dorsal and ventral body bends initiated at the anterior end of the animal $^{23}$ and feeds by rhythmic pharyngeal contractions called pumps ${ }^{24}$. When we overexpressed $n l p-22$ using an inducible heatshock promoter (Fig. 2a) during the normally active adult period, we observed quiescence of feeding and locomotion (Fig. 2b,c, Supplementary Movie 1, Supplementary Table S1). Two hours after heat shock, control animals made $15.9 \pm 7.3$ (all values presented as mean \pm s.d.) $(N=15)$ anterior body bends per min, whereas animals overexpressing $n l p-22$ made $0.55 \pm 1.1(N=25)$ body bends per min (Fig. 2b). Wild-type animals and transgenic control animals expressing GFP under the control of a heat-shock promoter pumped $2 \mathrm{~h}$ after heat shock at rates of $264.9 \pm 17.4$ $(N=20)$ and $273.6 \pm 17.5(N=20)$ pumps per min, respectively (Fig. 2c, Supplementary Table S1). Animals carrying the integrated or extrachromosomal Phsp16.2:nlp-22 arrays, qnIs142 or qnEx95, pumped at rates of $18.6 \pm 31.1 \quad(N=37)$ and $25.2 \pm 32.3(N=40)$ per min, respectively, $2 \mathrm{~h}$ after heat shock (Fig. 2c, Supplementary Table S1). In the absence of either the signal sequence or the dibasic residues predicted to be necessary for preproprotein cleavage ${ }^{25}, n l p-22$ overexpression no longer promoted behavioural quiescence (Fig. 2c), indicating that these features of the protein are important for its biological activity. When we mutated any of the four C-terminal amino acids of the predicted peptide, overexpression of the gene no longer caused quiescence (Fig. 2c), indicating that these four amino acids are important for the peptide's biological function. Nine hours after $n l p-22$ overexpression, all the animals resumed feeding, indicating that the reduction in behaviour was not due to an injurious effect (Fig. 2d).

NLP-22-induced quiescence resembles lethargus quiescence. During lethargus, animals are less responsive to stimuli, a property shared with sleeping vertebrates ${ }^{12}$. In addition, worms in lethargus move backwards more often than non-lethargus worms $^{26}$. Similar to wild-type worms in lethargus, adult worms overexpressing $n l p-22$ showed reduced responsiveness to optic or chemical stimulation compared with transgenic control animals that overexpressed GFP (Fig. 3a). Under mild heat-shock conditions, in which $n l p$-22-overexpressing adult animals were not completely quiescent, they moved backwards more often than control animals (Fig. 3b). Thus, overexpression of NLP-22 induces feeding and movement quiescence that is reminiscent of lethargus quiescence.

NLP-22 is required for proper quiescence during lethargus. Our overexpression studies demonstrate sufficiency of $n l p-22$ for the induction of behavioural quiescence. However, overexpression experiments are likely to result in supra-physiological a

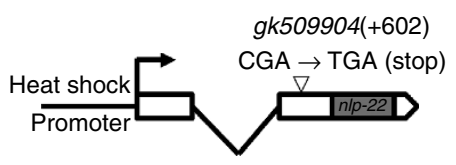

NLP-22 Preproprotein

Signal sequence (SS) Peptide

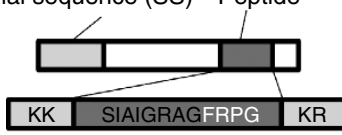

NLP-22 Peptide
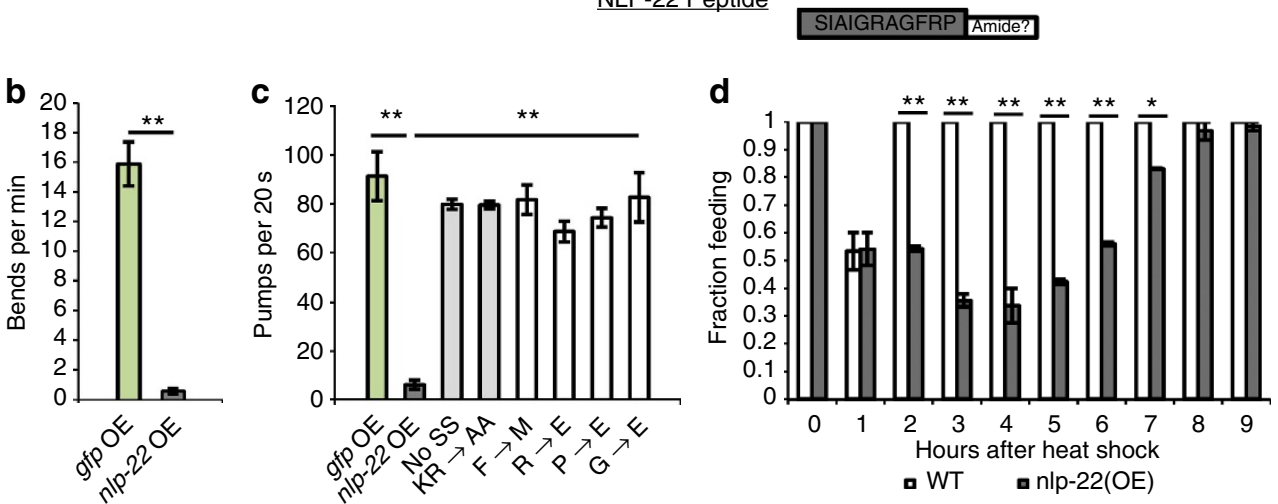

Figure 2 | NLP-22 induces behavioural quiescence. (a) Gene and protein structure of $n / p-22$ including overexpression construct. Animals show reduced movement (b) and feeding (c) relative to control animals overexpressing GFP (strain: TJ375), $2.5 \mathrm{~h}$ after $n / p-22$ induction. (c) Removing the signal sequence or mutating the KR or FRPG eliminates the ability of NLP-22 to reduce pumping rate ( ${ }^{\star} P<0.0001$, Student's two-tailed $t$-test, $N \geq 20$ ). (d) Animals overexpressing $n / p-22$ cease pumping, but recover $9 \mathrm{~h}$ after heat shock $\left({ }^{\star} P<0.001,{ }^{\star \star} P<0.0001\right.$, Fisher's exact test, $N \geq 20$ animals, two trials; Error bars represent s.e.m.). 

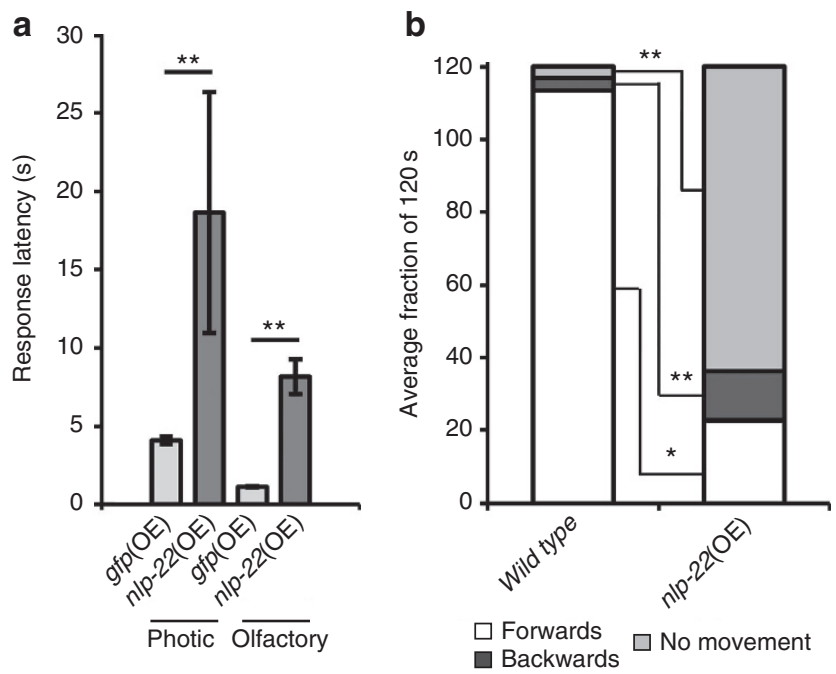

Figure 3 | Quiescence induced by nlp-22 OE resembles lethargus quiescence. (a) Animals that overexpress $n / p-22$ (strain: NQ251) are less responsive to chemical (1-octanol) and optical (blue light) stimulation than control animals that overexpress GFP (strain: TJ375) ${ }^{\star \star} P<0.0001$, Student's two-tailed $t$-test, $N \geq 20$; Error bars represent s.e.m.).

(b) Overexpressing $n / p-22$ via a mild heat shock, such that full quiescence is not induced, results in increased backwards movement. White, dark grey and light grey denote forwards, backwards and no movement, respectively. The average fraction of time spent moving forwards or moving backwards in a 120s window for $n / p-22$ overexpressing animals is significantly different from WT worms $\left({ }^{\star} P<0.001 .{ }^{\star \star} P<0.0001\right.$, Student's two-tailed t-test, $N=20$ ). In addition to the bias for backwards motion, $n / p-22$ overexpressing animals also moved significantly less than WT animals $\left({ }^{\star \star} P<0.0001\right.$, Student's two-tailed $t$-test, $\left.N=20\right)$.

concentrations of the peptide as well as spatially ectopic expression. Therefore, we proceeded to study a loss of function $n l p-22$ mutation to ask the question: Is $n l p-22$ necessary for quiescence? The $n l p$-22 allele $g k 509904$ has a point mutation that introduces a stop codon before the NLP-22 peptide (Fig. 2a); thus, it is likely to fully eliminate $n l p-22$ function. We measured quiescence in wildtype and $n l p-22(g k 509904)$ animals from the active L3 stage through development into adulthood. Total quiescence during L4 lethargus of $g k 509904$ mutants was reduced in comparison with total quiescence of wild-type animals (Fig. 4a,c and Supplementary Table S2). The quiescence defect of $g k 509904$ mutants was rescued by expression of a genomic fragment spanning the $n l p-22$ locus (Fig. 4a,c and Supplementary Table S2). To test for the functional relevance of the observed RIA gene reporter expression, we expressed $n l p-22$ double-stranded RNA (dsRNA) to knockdown $n l p-22$ mRNA specifically in RIA, and, as a control, in the hypodermis. Expression in the RIA neurons but not in the hypodermis reduced lethargus quiescence (Fig. 4b,c and Supplementary Table S2). In addition to reduced quiescence, $n l p-22$ (gk509904) mutants showed enhanced responsiveness to optic sensory stimulation during L4 lethargus quiescence bouts (Fig. 4d), demonstrating reduced sleep-like behaviour. Thus, $n l p-22$ is required for appropriate C. elegans sleep-like quiescence during lethargus.

NLP-22 signals through a PKA-dependent mechanism. To determine mechanisms by which $n l p-22$ induces sleep-like behaviour, we crossed animals carrying $n l p-22$ overexpression arrays into a subset of the genetic backgrounds that have been previously shown to affect C. elegans quiescence (kin-2 (ref. 27), egl-4 (ref. 12) and ceh-17 (ref. 14)) and into genetic backgrounds known to affect neuropeptide processing or release (egl-3 (ref. 28), egl-21 (ref. 29) and unc-31 (ref. 30)) (Supplementary Table S1). In all cases, but one, the quiescence-inducing effects of $n l p-22$ overexpression were no different in the mutant background from these effects in the wild-type background. The one observed exception was in kin-2(ce179) mutants, in which the normal inhibition of the catalytic subunits of the cAMP-dependent protein kinase PKA by the regulatory subunits is impaired due to a mutation in the regulatory subunit, resulting in excess activity of the catalytic subunit ${ }^{31}$. Excessive PKA activity causes increased wakefulness in C. elegans and other species ${ }^{32}$. kin-2 mutants are hyperactive ${ }^{33}$, are hyper-responsive to stimuli during lethargus, and show reduced lethargus quiescence ${ }^{27}$. In the kin-2-mutant background, Phsp16.2:nlp-22 transgenic animals continued to feed and move from 2.5 to $4.0 \mathrm{~h}$ after heat shock (Fig. 5a,b). Thus, a reduction in PKA activity is required for NLP-22-induced quiescence. Although kin-2 mutants continued to pump upon $n l p-22$ overexpression, pumping rates were reduced relative to kin-2-mutant controls (Supplementary Table S1), indicating that either residual kin-2 activity in the mutant analysed is sufficient to reduce PKA activity in response to $n l p-22$ overexpression or that there are also PKA-independent pathways regulating feeding and locomotion quiescence in response to $n l p-22$.

The RIAs have quiescence- and movement-promoting properties. Because the RIA neurons express the somnogenic peptide NLP-22, we hypothesized that they are quiescence-promoting neurons. To test this hypothesis, we used the strain VM1345, which expresses the cell death gene interleukin-l $\beta$-converting Enzyme $(I C E)^{34-36}$ in the RIA neurons. Because of the incomplete penetrance of the RIA ablation phenotype in VM1345, in a subset of the animals only one of the two RIAs died. We compared L4 lethargus quiescence in animals lacking one or two RIAs to a control transgenic strain with both RIA neurons intact. There was a significant reduction in lethargus quiescence in animals lacking both RIA neurons (Fig. 6a), consistent with the hypothesis that RIA neurons promote quiescence. Animals lacking only one of the RIA neurons also showed a small but significant reduction in L4 lethargus quiescence compared with control transgenic animals (Fig. 6a).

This neuron ablation experiment supports the notion that the RIA neurons promote sleep-like behaviour. However, removing the neuron by genetic ablation is a chronic manipulation that removes all functions of the neurons, including both those related to rapid signalling via membrane voltage changes as well as chronic signalling via mechanisms independent of membrane voltage. To test whether acute depolarization of the RIA neurons also induces quiescence, we made transgenic animals expressing the blue light-activated cation channel channelrhosopsin-2 $(\mathrm{ChR} 2)^{37}$ in the RIA neurons. We optogenetically depolarized the RIA neurons during the active L4 stage as well as during L4 lethargus. Animals carrying the ChR2 transgene in the RIA neurons were cultivated in the absence $(N=4)$ or presence $(N=7)$ of the ChR2 co-factor all-trans retinal (ATR) and followed for $8 \mathrm{~h}$ during the active L4 stage. The animals were exposed to blue light for $15 \mathrm{~s}$ every $30 \mathrm{~min}$ and monitored for locomotion and feeding quiescence. Stimulating the RIA neurons during the active L4 stage caused no induction in behavioural quiescence. In contrast, optogenetic depolarization of the RIA neurons during L4 lethargus had a locomotion-stimulating effect. Compared with control animals lacking the ChR2 transgene and with control animals carrying the transgene, but lacking ATR, stimulation of the RIA neurons increased movement during lethargus (Fig. 6b). Thus, acute depolarization of the RIA neurons during lethargus promotes wake-like behaviour. 
a

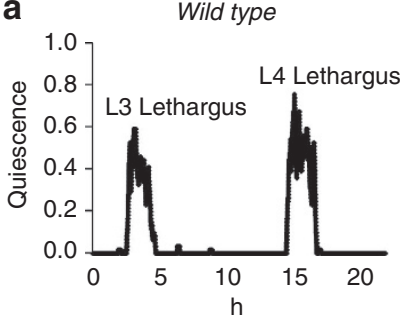

b
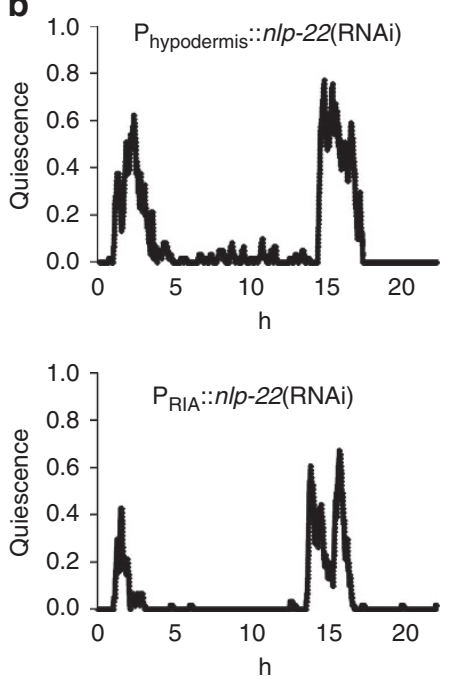
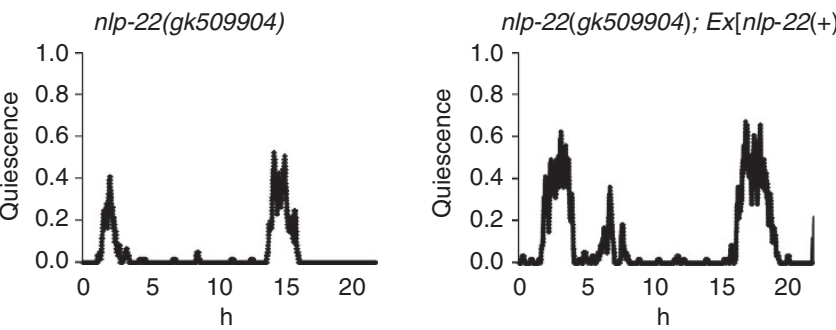

C

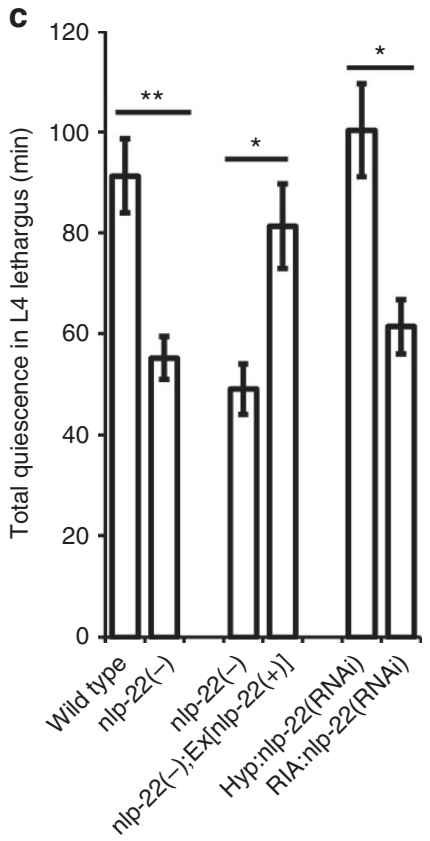

d

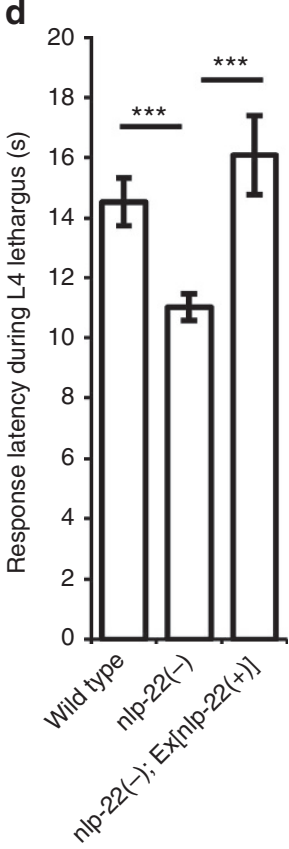

Figure 4 | $\mathbf{n l p - 2 2}$ is required for normal quiescence during lethargus. (a) Fraction of quiescence in a 10-min moving window in a wild-type animal, an $n / p-22$ (gk509904) mutant and an n/p-22(gk509904) mutant carrying an nlp-22 genomic rescuing transgene. The $x$-axis is hours from the start of recording in the late third larval stage. (b) Quiescence in animals expressing double-stranded nlp-22 RNA in the hypodermis and in the RIA neurons. Average quiescence $\left({ }^{\star} P<0.05,{ }^{\star \star} P<0.01,{ }^{\star \star \star} P<0.001\right.$, Student's two-tailed $t$-test; $N \geq 10$, error bars represent s.e.m.), (c) and response latencies to blue light (d) during fourth larval stage lethargus ( ${ }^{\star \star \star} P<0.001$, Student's two-tailed $t$-test; $N \geq 28$ animals, error bars represent s.e.m.).
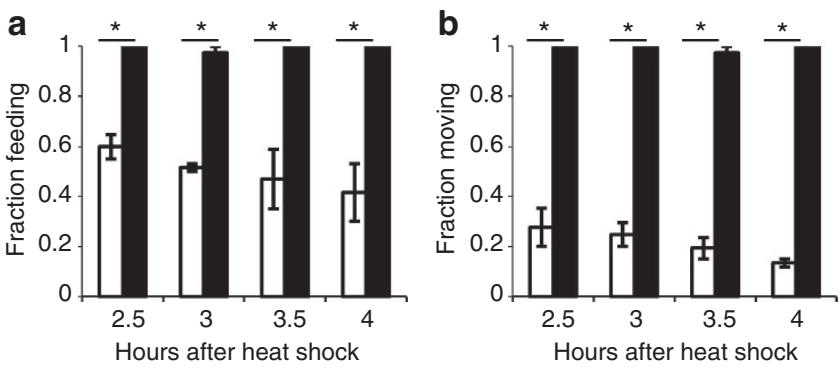

Figure 5 | NLP-22-induced quiescence requires the protein kinase A subunit KIN-2. Feeding (a) and locomotion (b) quiescence induced by $n / p-22$ overexpression is impaired in kin-2 mutants ( ${ }^{\star} P<0.0001$, Fisher's exact test, $N>20$ animals, 2 trials; error bars represent s.e.m.). Unfilled bars represent animals of genotype qnls142(Phsp-16.2:nlp-22;

Phsp-16.2:gfp; Pmyo-2:mCherry; unc-119( +$)$ ) and filled bars represent animals of genotype qn/s142; kin-2(ce179)X.

We conclude that the RIA neurons have both quiescencepromoting effects, revealed by chronic ablation, and wakepromoting effects, revealed by acute depolarization. Thus, the RIA neurons are complex regulators of lethargus quiescence with both movement- and quiescence-promoting roles.
NLP-22 is structurally similar to mammalian Neuromedin S. The NLP-22 predicted peptide has a glycine at its C-terminus. In previous analyses, C. elegans neuropeptides with a C-terminal glycine have been shown to be deglycinated and then amidated during peptide maturation ${ }^{38}$. Hence, NLP-22 has been termed an FRPamide peptide, corresponding to a Phenylalanine $(\mathrm{F})$ Arginine(R)-Proline(P) motif ${ }^{19}$ (Fig. 2a). Using the alignment program Muscle (version 3.7) ${ }^{39}$ on the Phylemon 2.0 platform $^{40}$, we found that the predicted NLP-22 neuropeptide in C. elegans is nearly identical to orthologous proteins of four other nematodes (Fig. 8a), indicating strong functional selection on the neuropeptide sequence. Comparing the NLP-22 neuropeptide with human neuropeptides, we noted similarities with neuromedin $\mathrm{S}$ (NMS). Both the NLP-22 and NMS peptides are located near the C-terminus of the preproprotein (Fig. 8b) and are composed of the dipeptide Glycine $(\mathrm{G})$-Arginine $(\mathrm{R})$ located in the middle of the peptide and the tripeptide FRP located at or close to the C-terminus (Fig. 8b).

To further compare NLP-22 to NMS, we used the peptide structure algorithm Pep-Fold ${ }^{41}$, which predicts the threedimensional structures of small peptides. Pep-Fold predicts that NLP-22 forms an anti-parallel beta-sheet where the conserved GR dipeptide is within the loop, and the C-terminal FRP is near the end of the second sheet (Fig. 8c). Interestingly, human NMS is predicted to form the same general structure (Fig. 8c). 

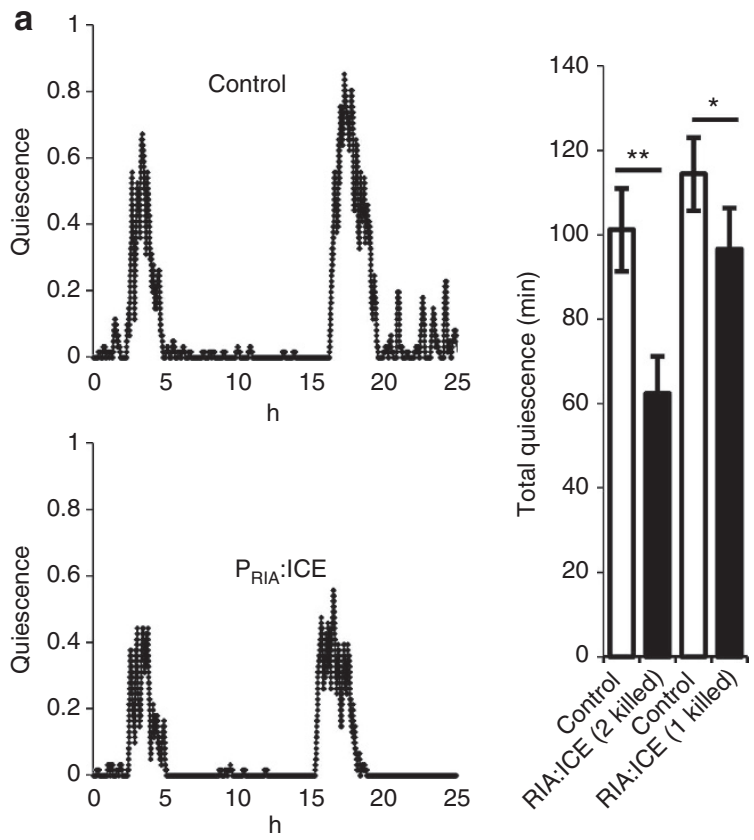

b

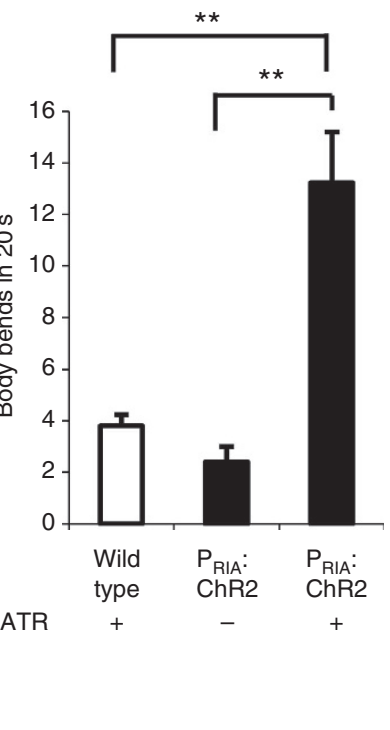

Figure 6 | The RIA neurons have both quiescence- and movement-promoting functions. (a) Genetic ablation of the RIA interneurons using the cell death protein ICE (strain:VM1345) decreases quiescence during lethargus compared with control transgenic animals (strain: NQ156). The traces depict the fraction of quiescence in a 10-min time interval. The top trace is of a control animal of genotype lin-15(n765ts)X; qnEx48(Pins-4:gfp:PEST; lin-15( + )), and the bottom trace is of an experimental animal of genotype lin-15(n765ts)X; akEx211(Pglr-3:gfp; Pglr-3:ICE; lin-15( + )). Total quiescence during L4 lethargus is lower in experimental animals lacking either one or both RIA neurons than in control animals to which they were paired $\left({ }^{\star} P<0.05\right.$, ${ }^{\star \star} P<0.001$, Student's two-tailed $t$-test, $N \geq 9$; error bars represent s.e.m.). (b) Optogenetic stimulation of the RIA neurons promotes movement during lethargus. WT animals

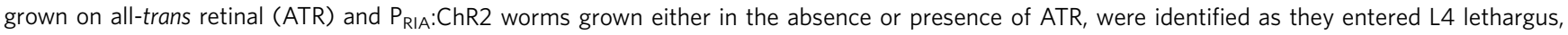
transferred to a new plate and were unperturbed for $15 \mathrm{~min}$ until they became quiescent. Body bends were counted for $1 \mathrm{~min}$ after a 15 -s exposure of quiescent animals to blue light. $P_{\text {RIA }}$ :ChR2 animals on ATR moved significantly more than control animals $\left({ }^{\star} P<0.05\right.$, ${ }^{\star \star} P<0.001$, Student's two-tailed t-test, WT $(N=15)$, No ATR vs ATR $(N \geq 20)$; error bars represent s.e.m.).

These structural similarities suggest that NLP-22 and NMS may be orthologous peptides.

\section{Discussion}

Wake-promoting neuropeptides such as hypocretin/orexin in vertebrates and PDF in Drosophila have well-defined roles as modulators of sleep/wake as an output of the circadian clock ${ }^{9}$. However, few physiologically relevant sleep-promoting peptides have been identified ${ }^{3,5,42}$, and their relationship to the circadian clock is poorly understood. We have identified NLP-22 as a C. elegans somnogenic peptide. NLP-22 activity is partially regulated at the level of mRNA abundance in response to a LIN-42/PERbased larval clock, suggesting that similar somnogens may function as the output to the PER-based circadian clock in other species.

How might LIN-42 be regulating nlp-22 expression? Drosophila PER acts as a cell autonomous negative regulator of transcription of genes that are positively regulated by the transcription factors CLK and $\mathrm{CYC}^{20}$. In response to ectopic expression of LIN-42, we observed nlp-22 mRNA induction only after $6-8 \mathrm{~h}$, which is delayed relative to a typical heat-shock response ${ }^{43}$. If LIN-42 were functioning in C. elegans similarly to PER function in Drosophila, then LIN-42 could repress transcription of a gene that negatively regulates $n l p-22$ expression in the same cell. Alternatively, the interaction could be non-cell autonomous. In support of a possible non-cell autonomous interaction, restoration of LIN-42 in the hypodermis of lin-42 mutants partially rescues the asynchronous nature of lethargus quiescence ${ }^{17}$ yet $n l p-22$ is expressed outside the hypodermis, in the RIA interneurons. Even if LIN-42 were regulating $n l p-22$ expression directly, then it must also regulate other genes, as $n l p-22$ mutants do not show other phenotypes observed for lin-42 mutants such as heterochronic defects, slow growth and moulting defects ${ }^{17,44}$.

What is the role of the RIA interneurons during lethargus quiescence? The RIAs are a highly connected pair of interneurons that are required for thermotaxis ${ }^{45}$ and aversive/associative learning ${ }^{35,46-48}$. Our data suggest that the RIAs also control sleep-like behaviour. Surprisingly, our experiments revealed both quiescence- and movement-promoting functions for the RIA neurons. Our results suggest that the RIAs release two factors with different behavioural effects: the neuropeptide NLP-22, which decreases PKA activity in an unidentified cell type (Fig. 7) to promote quiescent behaviour and an unknown transmitter, which promotes movement. As acute depolarization of RIA promotes movement and not quiescence, the mechanism of NLP22 release may be different from that of the movement-promoting transmitter. NLP-22 release may be regulated by secondary messengers independently from the membrane voltage. For example, a local calcium increase without membrane potential change could promote NLP-22 secretion without affecting the release of wake-promoting factor. Consistent with this possibility, the RIA neurons show subcellular compartmentalization of intracellular calcium levels ${ }^{49}$. In addition, NLP-22 availability is regulated at least partially at the level of mRNA abundance. Regardless of the explanation for these results, they highlight both the limitation of relying on ablation alone to infer neuron function and the benefit of using acute optogenetic activation to understand neuronal function.

We have shown that NLP-22 and mammalian NMS are structurally similar (Fig. 8). In addition, NLP-22 and NMS show 
intriguing functional similarities. NMS shows a diurnal pattern of mRNA expression and is predominately expressed in a small set of central nervous system neurons in the suprachiasmatic nucleus ${ }^{7}$. Like NMS, NLP-22 cycles its expression, only as the output of a larval-based clock, and like NMS, NLP-22 is selectively expressed, in a single-neuron pair, the RIAs (Fig. 1c). Although the suprachiasmatic nucleus is well known as the master circadian clock pacemaker ${ }^{50}$, a role for the RIAs in regulating the analogous larval clock in C. elegans has not been reported. The RIAs are required for $C$. elegans memory behaviours ${ }^{35,48}$, behaviours that in mammals are sensitive to the sleep-wake history of the animal ${ }^{51}$.

The central nervous system administration of NMS into mammals and birds inhibits feeding behaviour ${ }^{52-55}$. NLP-22 overexpression causes an almost complete block of feeding in

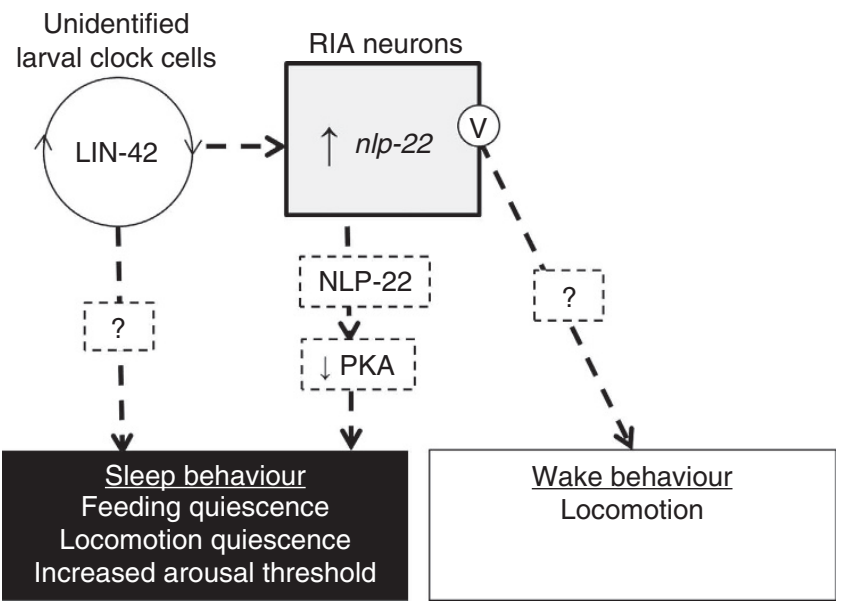

Figure 7 | Model for role of nIp-22 and RIA in sleep-like quiescence regulation. LIN-42 functions in as yet unidentified larval clock cells to regulate nlp-22 expression as well as other quiescence-regulatory factors. Release of NLP-22 neuropeptide promotes sleep-like behaviour via a reduction in protein kinase $A$ (PKA) activity. RIA membrane depolarization (marked with the letter $\mathrm{V}$ ) results in the release of an unidentified wakepromoting neurotransmitter.
C. elegans (Fig. 2). The central nervous system administration of NMS in rats causes an inhibition of locomotion and a phase shift of the circadian behavioural rhythm ${ }^{7}$. NLP-22 overexpression causes behavioural quiescence during the normally active adult stage (Fig. 2). Our work predicts effects of NMS on sleep behaviour, but to our knowledge this has not yet been tested.

Although there are functional and structural similarities between NLP-22 and NMS, there are also clear differences. Mammalian NMS has a highly conserved C-terminal RN motif that is not present in C. elegans NLP-22. When we overexpressed human NMS(17-33), which contains the 17 C-terminal amino acids of NMS including the amino acids conserved with NLP-22 and which is predicted to form a similar three-dimensional structure as NLP-22 and full-length NMS (Supplementary Fig. S2a), we did not observe behavioural quiescence in C. elegans (Supplementary Fig. S2b). Furthermore, we could not identify genes coding for proteins predicted to be processed into NLP-22 or NMS-like peptides in the genomes of insects or fish. Finally, loss of function of each of four G-protein-coupled receptors that are weakly similar to a mammalian NMS receptor ${ }^{56}$ did not suppress the NLP-22-induced quiescence (Supplementary Table S1). Neuropeptides and neuropeptide receptors often show coevolution, leading to difficulty in identifying homologues across phyla ${ }^{57}$. Thus, future additional data, including the identification of the NLP-22 post-synaptic signalling pathway, will be required to determine whether NLP-22 and NMS are true homologues.

In summary, our work defines the first role for a somnogenic neuropeptide in $C$. elegans and identifies a pair of neurons, the RIAs, in the regulation of $C$. elegans sleep-like behaviour. Although C. elegans sleep-like behavioural research is still in its nascent stage, the simplicity of this model animal's nervous system combined with recent progress in the identification of cells involved in sleep-like behavioural regulation ${ }^{13-15,58,59}$ holds promise for rapid future progress in delineating the genes and circuits regulating sleep and wake.

\section{Methods}

Cultivation methods and strains. Animals were cultivated on NGM agar and, unless noted otherwise, were fed the OP50 E. coli derivative strain DA837 (ref. 60). All experiments were performed on hermaphrodites. The following strains were used in this study: N2 (Bristol), EG4322 ttTi5605 II; unc-119(ed3)III, TJ375

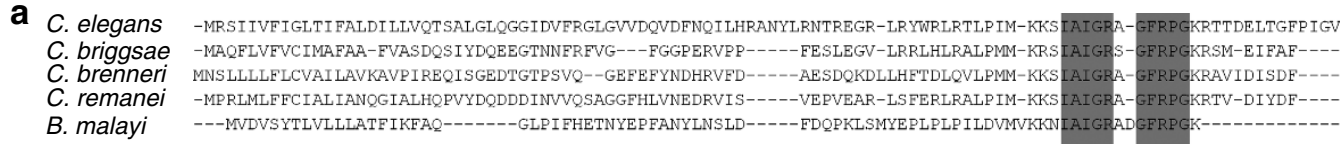

b

C. elegans NLP-22
H. sapiens NMS
M. musclus NMS
R. norvegicus NMS
B. orientalis NMS

B. orientalis NMS

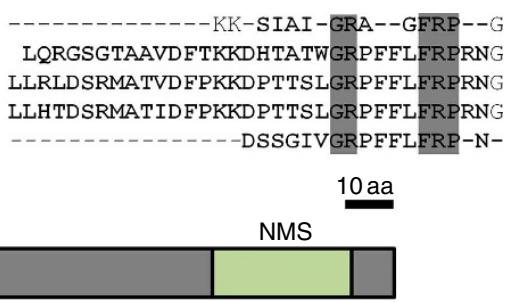

NLP-22

\begin{tabular}{|c|l|l|l|}
\hline $\begin{array}{c}\text { Signal } \\
\text { sequence }\end{array}$ & & & \\
\hline
\end{tabular}

C NLP-22

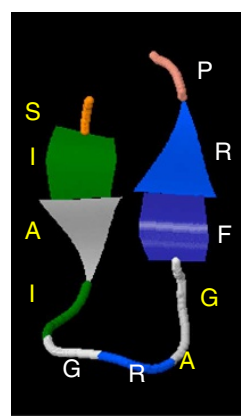

H. sapien - NMS

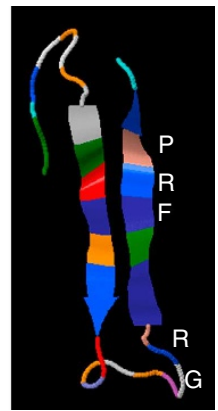

Figure 8 | NLP-22 is similar to Neuromedin S. (a) Alignment of NLP-22 preproprotein with orthologs from four nematode species. High conservation (grey box) is observed in the predicted neuropeptide sequence. (b) Amino-acid sequence similarities (grey boxes) between NLP-22 and vertebrate Neuromedin S peptides. The preproprotein structure of human Neuromedin S and NLP-22 is also shown. Each has an N-terminal signal sequence and a single, C-terminal peptide. The preproprotein is drawn to scale (Bar $=10$ amino acids). (c) Predicted structures of NLP-22 and of NMS. The conserved GR dipeptide and FRP tripeptide motifs are shown in white. 
gpIs1(Phsp-16.2::gfp), NQ156 lin-15(n765ts)X; qnEx48(Pins-4:gfp:PEST; lin15(+)), NQ216 unc-119(ed3)III;qnEx95(Phsp-16.2::nlp-22; Pmyo-2:mCherry; unc$119(+))$, IB16 ceh-17(np1)I, NQ230 ceh-17(np1)I; qnEx95, NQ235 nmur 4(ok1381)I; qnEx95, NQ250 qnIs101(Pnlp-22:nlp-22:gfp; Pmyo-2:mCherry; unc119(+)), NQ251 qnIs142(Phsp-16.2:nlp-22; Phsp-16.2:gfp; Pmyo-2:mCherry, unc $119(+))$, DA509 unc-31(e928)IV, NQ256 unc-31(e928)IV;qnEx95, NQ266 unc119(ed3)III;qnEx128(Pnlp-22:gfp:nlp-22; Pmyo-2:mCherry; unc-119(+)), NQ269 unc-119(ed3)III;qnEx131(Phsp-16.2:nlp-22(No Signal Sequence); Pmyo-2:mCherry; unc-119(+)), NQ270 unc-119(ed3)III;qnEx132(Phsp-16.2:nlp-22(No Signal Sequence); Pmyo-2:mCherry; unc-119(+)), NQ274 nmur-1(ok1387)X; qnEx95, NQ275 nmur-3(ok2295)X; qnEx95, NQ282 unc-119(ed3)III;qnEx139(Phsp16.2:nlp-22(No Signal Sequence); Pmyo-2:mCherry; unc-119(+)), NQ285 nmur2(ok3502)II; qnEx95, NQ305 sid-1(pk3321)V; qnIs137(Pdpy-7:nlp-22(RNAi); Pmyo2:mCherry; unc-119(+)), NQ314 unc-119(ed3)III; qnEx160(Phsp-16.2:nlp22(KR $\rightarrow$ AA); Pmyo-2:mCherry; unc-119(+)), KP1873 egl-3(nu349) V, NQ319 egl-3(nu349)V; qnEx95, KP2018 egl-21(n476)IV, NQ320 egl-21(n476)IV;qnEx95, MT1074 egl-4(n479)IV, NQ321 egl-4(n479)V;qnEx95, NQ333 unc-119(ed3)III; qnEx162(Pnlp-22:Intron: gfp; Pmyo-2:mCherry; unc-119(+)), NQ367 unc119(ed3)III; qnEx178(Phsp-16.2:nlp-22(KR $\rightarrow$ AA); Pmyo-2:mCherry; unc-119(+)), NQ374 unc-119(ed3)III; qnEx185(Phsp-16.2:nlp-22(FRPG $\rightarrow$ MRPG); Pmyo2:mCherry; unc-119(+)), NQ375 unc-119(ed3)III;qnEx186(Phsp-16.2:nlp22(FRPG $\rightarrow$ MRPG); Pmyo-2:mCherry; unc-119(+)), NQ376 sid-1(pk3321)V; qnIs157(glr-3:nlp-22(RNAi); myo-2:mCherry; unc-119(+)), NQ378 unc119(ed3)III;qnEx187(Phsp-16.2:nlp-22(FRPG $\rightarrow$ FEPG); Pmyo-2:mCherry; unc119(+)), NQ379 unc-119(ed3)III;qnEx187(Phsp-16.2:nlp-22(FRPG $\rightarrow$ FEPG); Pmyo-2:mCherry; unc-119(+)), NQ383 unc-119(ed3)III; qnEx192(Phsp-16.2:nlp22(FRPG $\rightarrow$ FRPE); Pmyo-2:mCherry; unc-119(+)), NQ392 unc119(ed3)III;qnEx188(Phsp-16.2:nlp-22(FRPG $\rightarrow$ FREG); Pmyo-2:mCherry; unc119(+)), NQ393 unc-119(ed3)III;qnEx189(Phsp-16.2:nlp-22(FRPG $\rightarrow$ FREG); Pmyo-2:mCherry; unc-119(+)), NQ448 unc-119(ed3)III; qnEx224(Pglr3:ChR2::YFP (Regular) + unc-122:gfp + unc-119(+)) ARF240 aaaIs1(Phsp-16::lin42a::unc-54utr; Pmyo-2:dsRed), NQ596 nlp-22(gk509904)X, NQ603 nlp22(gk509904)X; qnEx311(nlp-22(+); Pmyo-2:Cherry), KG532 kin-2(cd179)X, NQ606 kin-2(cd179)X; qnIs142; VC40199 nlp-22(gk509904)X; NQ667 kin2(ce179)X; qnEx95, NQ668 egl-4(n479)IV; qnIs142, NQ670 qnEx95, VM1345 lin-15(n765ts)X; akEx211(Pglr-3:gfp; Pglr-3:ICE; lin-15(+)), RB1288 nmur1(ok1387)X, RB2526 nmur-2(ok3502)II, VC1974 nmur-3(ok2295)X, RB1284 nmur-4(ok1381)I.

Molecular biology and transgenesis. All DNA constructs were made using overlap-extension $\mathrm{PCR}^{61}$. In brief, to fuse two or more DNA fragments, we amplified (Herculase Enhanced DNA Polymerase, Agilent Technologies) the individual DNA fragments with oligonucleotides that included $5^{\prime}$-extensions complementary to the fusion target. Individually amplified DNA fragments were then mixed without primers in the presence of DNA polymerase in order to anneal and extend the fused product. The fused product was purified (QIAquick PCR Purification Kit, Qiagen) and amplified using nested primers. The size of the final PCR product was verified by performing gel electrophoresis ${ }^{61}$. Oligonucleotides used for making these constructs are listed in Supplementary Table S3. For $n l p-22$ overexpression, the promoter of $h s p-16.2$ was amplified from the vector pPD49.83 (obtained from Addgene) and fused to the genomic sequence of $n l p-22(+1$ to $+1,451$ ) (where nucleotide number refers to the nucleotide position relative to the start site of translation). Both the extrachromosomal array, qnEx95, and the integrated array, qnIs142, were analysed in each behavioural assay. The mutated $n l p-22$ overexpression constructs (No signal sequence (SS), KR $\rightarrow$ AA, FRPG $\rightarrow$ MRPG, FRPG $\rightarrow$ FEPG, FRPG $\rightarrow$ FREG, FRPG $\rightarrow$ FRPE) were created by introducing the desired mutations by overlap-extension PCR using oligonucleotide $5^{\prime}$-extensions that encoded the amino-acid changes ${ }^{61}$. Each mutated fused PCR product was cloned into a pCR2.1-TOPO TA Vector (Invitrogen) and then sequenced to verify that the intended mutation but no other mutation was introduced. At least two and often three independent lines were analysed for each behavioural analysis. To make a fusion protein in which GFP is at the C-terminus of the NLP-22 protein, the genomic sequence of $n l p-22(-450$ to +701$)$ was fused in frame to $g f p$ followed by the $u n c-543^{\prime} \mathrm{UTR}$, which was amplified from the vector pPD95.75 (Addgene). To make a fusion in which GFP is at the $\mathrm{N}$-terminus of the NLP-22 protein, the promoter $(-450$ to +3$)$ of $n l p-22$ was fused to the coding sequence of GFP amplified from pPD95.75 only excluding the stop codon and fused in frame to the genomic sequence of $n l p-22(+1$ to +701$)$, which included the $n l p-223^{\prime} \mathrm{UTR}$. The transcriptional reporter for $n l p-22$ (Pnlp-22:Intron:gfp) was made by fusing the promoter of $n l p-22(-450$ to +3$)$ to the coding sequence of GFP, amplified from pPD95.75 and replacing one of the synthetic introns of $g f p$ with the intron of $n l p-22(+85$ to +481$)$. To drive expression of $n l p-22$ dsRNA in the RIA neurons, the promoter of $g l r-3(-5,500$ to -1$)$ was fused to the genomic sequence of $n l p-22$ spanning the region +13 to +653 in the sense orientation, and, in a separate reaction, to the $n l p-22$ genomic sequences spanning the same nucleotides in the anti-sense orientation. These constructs lack a start codon and $3^{\prime} \mathrm{UTR}$ of $n l p-22$. To drive expression of $n l p-22 \mathrm{dsRNA}$ in the hypodermis, the promoter of $d p y-7$ ( -417 to -1 ) amplified from $\mathrm{N} 2$ genomic DNA was fused to $n l p-22$ sense and anti-sense DNA. The genomic rescuing fragment of $n l p-22$ spanned from -450 to $+1,451$ relative to the $n l p-22$ start codon. To overexpress
Neuromedin S (17-33) (Phoenix Pharmaceuticals), we replaced the coding sequence for the $n l p-22$ peptide with the coding sequence for Neuromedin $S$ (17-33) and fused the nlp-22-NMS(17-33) chimeric sequence to the heat-shock promoter.

Transgenic animals were created by microinjection ${ }^{62}$ using a Leica DMIRB inverted DIC microscope equipped with an Eppendorf Femtojet microinjection system. EG4322 animals were injected with $25-50 \mathrm{ng}^{-1} \mathrm{l}^{-1}$ of each construct in combination with $5 \mathrm{ng}^{-1} \mathrm{l}^{-1}$ pCFJ90 (Pmyo-2:mCherry), with $50-100 \mathrm{ng}^{-1}$ of Punc-122:gfp or pCFJ104 (Prab-3::mCherry) and pCFJ151 (unc-119(+)) up to a total concentration of $150 \mathrm{ng} \mu \mathrm{l}^{-1}$. To make the $n l p-22$ RNAi strains, $25 \mathrm{ng} \mu \mathrm{l}^{-1}$ of $n l p-22$ (sense) and $n l p$-22(anti-sense) were injected together with the transgenesis markers pCFJ90 and pCFJ151. For behavioural experiments using transgenic animals carrying extrachromosomal arrays, at least two distinct lines were analysed. In most cases, quantitative behavioural analysis was performed only on one of these transgenic lines, but in all cases the other line(s) gave the same qualitative result.

The integrated transgenes qnIs101,137, 142 and 157 were constructed by UV irradiation of the extrachromosomal transgenes qnEx101, 137, 142 and 157 (ref. 63). A mix of $\sim 100 \mathrm{~L} 4$ and adult transgenic animals were placed in a Spectrolinker XL-1500 UV Crosslinker (Spectronics Corporation) and exposed to $30,000 \mu \mathrm{J} \mathrm{cm}^{-2}$ of ultraviolet light. Irradiated animals were plated at a density of five animals per plate and grown for multiple generations. Integrants were identified as those that produced $100 \%$ transgenic progeny. Each integrated strain was out-crossed to the wild-type strain a minimum of three times before analysis

The strain VC40199 containing the $n l p-22(g k 509904) \mathrm{X}$ allele was produced by the million mutation project ${ }^{64}$ and obtained from the Caenorhabditis Genetics Consortium. The CGA to TGA mutation in $n l p-22$ reported for this strain was verified by sequencing a genomic PCR fragment, which was PCR-amplified using the primers oNQ552 and oNQ534 (Supplementary Table S3). The strain NQ596 $n l p-22$ (gk509904) X was generated by crossing the strain VC40199 to N2 males and then crossing resultant males back to VC40199. This procedure was repeated four times. A twitcher phenotype, which was present in VC40199 animals, was not present in the out-crossed NQ596 animals.

Overexpression analysis. For all overexpression experiments, animals carrying arrays with inducible heat-shock promoters were grown to young adulthood at $20^{\circ} \mathrm{C}$, transferred to the surface of NGM agar 6-cm diameter plates fully seeded with a lawn of DA837 E. coli bacteria, wrapped in Parafilm and immersed in a $33^{\circ} \mathrm{C}$ water bath for $30 \mathrm{~min}$. Heat-shocked animals were placed at $20^{\circ} \mathrm{C}$ before analysis. Pharyngeal pumping and body bends were counted during $20 \mathrm{~s}$ and $1 \mathrm{~min}$ intervals, respectively, two hours after heat-shock. Arousal threshold was determined by measuring the latency to respond to exposure to blue light ${ }^{65}$ or exposure to a hair dipped in a freshly prepared mixture of $30 \%$ octanol and $70 \%$ ethanol ${ }^{66}$. Response to octanol was defined as backwards movement of the magnitude corresponding to one-third of the worm's length. Response to blue light was defined as movement (either forward or backwards) of magnitude equal to one-half of the animal's body length. Arousal threshold assays of $n l p-22$ mutants and $n l p-22$ mutants carrying a rescuing transgene were performed by an investigator who was blinded to genotype. The blinding was set up by a colleague without the use of a computer randomization program. It was impossible to blind the investigator to the genotype of the $n l p-22(\mathrm{OE})$ transgenic animals because these animals were extremely quiescent and retained excessive eggs.

In experiments designed to assess the effects of mild overexpression of $n l p-22$ on direction of locomotion, animals were analysed $1 \mathrm{~h}$ after a $15 \mathrm{~min} 33^{\circ} \mathrm{C}$ heat exposure. Behaviour was assessed on an agar surface devoid of bacteria. During a 120 -s period, we measured with a hand-held timer the total time spent moving forwards as well as the total time spent moving backwards. The average of 20 experimental animals expressing Phsp16.2:nlp-22 (qnIs142) was compared with the average of 20 control $\mathrm{N} 2$ animals.

Imaging of fluorescence. For GFP and differential interference contrast imaging, animals were mounted on $5 \%$ agar pads, immobilized with $15 \mathrm{mM}$ levamisole and observed through a $\times 63$ or $\times 100$ objective lens on a Leica DM5500B microscope. Leica LAS software was used to capture images.

Quiescence measurements. For automated measurements of lethargus quiescence, we placed single larva in moulded, concave polydimethylsiloxane (PDMS) wells of diameter $3 \mathrm{~mm}$ and depth $2.5 \mathrm{~mm}$, filled with $15 \mu \mathrm{l}$ of NGM agar and seeded with DA837 bacteria. In each experiment, we placed one experimental and one control animal into two adjacent wells, and the PDMS was then placed on a Diagnostics Instruments microscope base and illuminated for bright-field microscopy, using white light supplied to the base with a fibre optic cable from a Schott DCR III light source. A camera $(659 \times 494$ pixels, scA640-70fm, Basler Vision Technologies) mounted on a Zeiss Stemi 2000 stereomicroscope captured an image with an 8-bit grayscale of both wells every $10 \mathrm{~s}$. At this magnification and our camera acquisition setting, the spatial resolution was 12.5 micrometers per pixel. To quantify quiescence, we monitored animals for time a period between 12 and $48 \mathrm{~h}$ and used a machine vision frame subtraction principle to identify 10 -s epochs of behavioural quiescence ${ }^{12,27,67}$. Recordings were performed in an environmental 
room at $19 \pm 2{ }^{\circ} \mathrm{C}$. In comparison with conditions used in previous quiescence measurements, the room temperature was lower and pixel resolution was reduced, leading to quiescence measurements that were greater than those previously reported $^{12}$. For each experimental condition, quiescence of at least six animals was measured because we previously found this number to be sufficient to detect a difference in quiescence ${ }^{12}$. For most conditions, 9-11 animals were used. Data were discarded if the temperature of the room exceeded $21^{\circ} \mathrm{C}$ due to equipment malfunction or if one of the two worms burrowed in the agar. Quiescence data from VM1345 were discarded when, upon inspection with epifluorescence, we determined that neither RIA had died. We censored 12\% of the VM1345 data for that reason.

qPCR. L2 or L3 worms were picked to fresh plates seeded with DA837 bacteria and allowed to enter lethargus. We identified worms in L2 or L3 lethargus, using a Leica MZ16 stereomicroscope, by an absence of locomotion and feeding. A subset of worms was collected for RNA isolation in either L2 or L3 lethargus, whereas the remaining animals were allowed to mature for additional durations before collecting. The experiment was performed at $25^{\circ} \mathrm{C}$ to speed development. L4 lethargus animals were similarly identified by first transferring mid L4 animals to a fresh agar dish seeded with DA837 and then collecting animals that had stopped moving and feeding.

To test lin-42-dependent induction of nlp-22 mRNA, ARF240 first day adult animals, which were cultivated at $15^{\circ} \mathrm{C}$, were submersed while housed on an NGM agar surface fully seeded with bacteria in a $33^{\circ} \mathrm{C}$ water bath for $30 \mathrm{~min}$ and then recovered at $25^{\circ} \mathrm{C}$. Control N2 animals were treated identically. RNA was collected before heat-shock treatment and at 4,6 and $8 \mathrm{~h}$ after the end of the heat treatment.

Total RNA was collected from each population using an RNAeasy mini kit (Qiagen), and cDNA was synthesized using the SuperScript one-step RT-PCR system (Invitrogen). We performed three or more biological replicates in each experiment, and for each biological replicate we used the average of two technical real-time PCR replicates. Real-time PCR was performed using Taqman Gene Expression Mastermix on an Applied Biosystems 7500 platform at the core services within the Penn Center for AIDS Research, an NIH-funded program (P30 AI 045008). Oligonucleotides used for the real-time PCR analysis were purchased from IDT, and their sequences are shown in Supplementary Table S3. Relative mRNA was determined by the delta-delta method ${ }^{68}$ by normalization to the expression of the gene pmp-3 (ref. 69).

\section{References}

1. Lin, L. et al. The sleep disorder canine narcolepsy is caused by a mutation in the hypocretin (orexin) receptor 2 gene. Cell 98, 365-376 (1999).

2. Cheng, M. Y. et al. Prokineticin 2 transmits the behavioural circadian rhythm of the suprachiasmatic nucleus. Nature 417, 405-410 (2002).

3. Kramer, A. et al. Regulation of daily locomotor activity and sleep by hypothalamic EGF receptor signaling. Science 294, 2511-2515 (2001).

4. Xu, Y. L. et al. Neuropeptide S: a neuropeptide promoting arousal and anxiolytic-like effects. Neuron 43, 487-497 (2004).

5. Lin, S. H. et al. Prolactin-releasing peptide (PrRP) promotes awakening and suppresses absence seizures. Neuroscience 114, 229-238 (2002).

6. Huitron-Resendiz, S. et al. Urotensin II modulates rapid eye movement sleep through activation of brainstem cholinergic neurons. J. Neurosci. 25, 5465-5474 (2005).

7. Mori, K. et al. Identification of neuromedin $\mathrm{S}$ and its possible role in the mammalian circadian oscillator system. EMBO J. 24, 325-335 (2005).

8. Parisky, K. M. et al. PDF cells are a GABA-responsive wake-promoting component of the Drosophila sleep circuit. Neuron 60, 672-682 (2008).

9. Crocker, A. \& Sehgal, A. Genetic analysis of sleep. Genes Dev. 24, 1220-1235 (2010).

10. Renn, S. C., Park, J. H., Rosbash, M., Hall, J. C. \& Taghert, P. H. A pdf neuropeptide gene mutation and ablation of PDF neurons each cause severe abnormalities of behavioral circadian rhythms in Drosophila. Cell 99, 791-802 (1999).

11. Singh, R. N. \& Sulston, J. E. Some observations on molting in Caenorhabditis elegans. Nematologica 24, 63-66 (1978).

12. Raizen, D. M. et al. Lethargus is a Caenorhabditis elegans sleep-like state. Nature 451, 569-572 (2008).

13. Driver, R. J., Lamb, A. L., Wyner, A. J. \& Raizen, D. M. DAF-16/FOXO regulates homeostasis of essential sleep-like behavior during larval transitions in C. elegans. Curr. Biol. 23, 501-506 (2013).

14. Van Buskirk, C. \& Sternberg, P. W. Epidermal growth factor signaling induces behavioral quiescence in Caenorhabditis elegans. Nat. Neurosci. 10, 1300-1307 (2007).

15. Choi, S., Chatzigeorgiou, M., Taylor, K. P., Schafer, W. R. \& Kaplan, J. M. Analysis of NPR-1 reveals a circuit mechanism for behavioral quiescence in C. elegans. Neuron 78, 869-880 (2013).

16. Jeon, M., Gardner, H. F., Miller, E. A., Deshler, J. \& Rougvie, A. E. Similarity of the C. elegans developmental timing protein LIN-42 to circadian rhythm proteins. Science 286, 1141-1146 (1999).
17. Monsalve, G. C., Van Buskirk, C. \& Frand, A. R. LIN-42/PERIOD controls cyclical and developmental progression of C. elegans molts. Curr. Biol. 21, 2033-2045 (2011).

18. Konopka, R. J. \& Benzer, S. Clock mutants of Drosophila melanogaster. Proc. Natl Acad. Sci. USA 68, 2112-2116 (1971).

19. Nathoo, A. N., Moeller, R. A., Westlund, B. A. \& Hart, A. C. Identification of neuropeptide-like protein gene families in Caenorhabditiselegans and other species. Proc. Natl Acad. Sci. USA 98, 14000-14005 (2001).

20. Dunlap, J. C. Molecular bases for circadian clocks. Cell 96, 271-290 (1999).

21. Gissendanner, C. R., Crossgrove, K., Kraus, K. A., Maina, C. V. \& Sluder, A. E. Expression and function of conserved nuclear receptor genes in Caenorhabditis elegans. Dev. Biol. 266, 399-416 (2004).

22. Hu, Z., Pym, E. C., Babu, K., Vashlishan Murray, A. B. \& Kaplan, J. M. A neuropeptide-mediated stretch response links muscle contraction to changes in neurotransmitter release. Neuron 71, 92-102 (2011).

23. Hart, Anne C., ed. Behavior (July 3, 2006), WormBook, ed. The C. elegans Research Community, WormBook, doi/10.1895/wormbook.1.87.1, http://www. wormbook.org.

24. Raizen, D., Song, B., Trojanowski, N. \& You, Y. Methods for measuring pharyngeal behaviors (December 18, 2012), WormBook, ed. The C. elegans Research Community, WormBook, doi/10.1895/wormbook.1.154.1, http://www.wormbook.org.

25. Li, C. \& Kim, K. Neuropeptides (September 25, 2008), WormBook, ed. The C. elegans Research Community, WormBook, doi/10.1895/wormbook.1.142.1, http://www.wormbook.org.

26. Iwanir, S. et al. The microarchitecture of $C$. elegans behavior during lethargus: homeostatic bout dynamics, a typical body posture, and regulation by a central neuron. Sleep 36, 385-395 (2013).

27. Belfer, S. J. et al. Caenorhabditis-in-drop array for monitoring C. elegans quiescent behavior. Sleep 36, 689-698G (2013).

28. Husson, S. J., Clynen, E., Baggerman, G., Janssen, T. \& Schoofs, L. Defective processing of neuropeptide precursors in Caenorhabditis elegans lacking proprotein convertase 2 (KPC-2/EGL-3): mutant analysis by mass spectrometry. J. Neurochem. 98, 1999-2012 (2006).

29. Husson, S. J. et al. Impaired processing of FLP and NLP peptides in carboxypeptidase E (EGL-21)-deficient Caenorhabditis elegans as analyzed by mass spectrometry. J. Neurochem. 102, 246-260 (2007)

30. Speese, S. et al. UNC-31 (CAPS) is required for dense-core vesicle but not synaptic vesicle exocytosis in Caenorhabditis elegans. J. Neurosci. 27, 6150-6162 (2007).

31. Schade, M. A., Reynolds, N. K., Dollins, C. M. \& Miller, K. G. Mutations that rescue the paralysis of Caenorhabditis elegans ric-8 (synembryn) mutants activate the $\mathrm{G}$ alpha(s) pathway and define a third major branch of the synaptic signaling network. Genetics 169, 631-649 (2005).

32. Zimmerman, J. E., Naidoo, N., Raizen, D. M. \& Pack, A. I. Conservation of sleep: insights from non-mammalian model systems. Trends. Neurosci. 31, 371-376 (2008).

33. Charlie, N. K., Schade, M. A., Thomure, A. M. \& Miller, K. G. Presynaptic UNC-31 (CAPS) is required to activate the G alpha(s) pathway of the Caenorhabditis elegans synaptic signaling network. Genetics 172, 943-961 (2006).

34. Yuan, J., Shaham, S., Ledoux, S., Ellis, H. M. \& Horvitz, H. R. The C. elegans cell death gene ced-3 encodes a protein similar to mammalian interleukin-1 beta-converting enzyme. Cell 75, 641-652 (1993).

35. Stetak, A., Horndli, F., Maricq, A. V., van den Heuvel, S. \& Hajnal, A. Neuron-specific regulation of associative learning and memory by MAGI-1 in C. elegans. PLoS One 4, e6019 (2009).

36. Miura, M., Zhu, H., Rotello, R., Hartwieg, E. A. \& Yuan, J. Induction of apoptosis in fibroblasts by IL-1 beta-converting enzyme, a mammalian homolog of the C. elegans cell death gene ced-3. Cell 75, 653-660 (1993).

37. Nagel, G. et al. Light activation of channelrhodopsin-2 in excitable cells of Caenorhabditis elegans triggers rapid behavioral responses. Curr. Biol. 15, 2279-2284 (2005).

38. Husson, S. J., Clynen, E., Baggerman, G., De Loof, A. \& Schoofs, L. Discovering neuropeptides in Caenorhabditis elegans by two dimensional liquid chromatography and mass spectrometry. Biochem. Biophys. Res. Commun. 335, 76-86 (2005).

39. Edgar, R. C. MUSCLE: multiple sequence alignment with high accuracy and high throughput. Nucleic Acids Res. 32, 1792-1797 (2004).

40. Sanchez, R. et al. Phylemon 2.0: a suite of web-tools for molecular evolution, phylogenetics, phylogenomics and hypotheses testing. Nucleic Acids Res. 39, W470-W474 (2011).

41. Maupetit, J., Derreumaux, P. \& Tuffery, P. PEP-FOLD: an online resource for de novo peptide structure prediction. Nucleic Acids Res. 37, W498-W503 (2009).

42. Jewett, K. A. \& Krueger, J. M. Humoral sleep regulation; interleukin-1 and tumor necrosis factor. Vitam. Horm. 89, 241-257 (2012). 
43. Candido, E. P. The small heat shock proteins of the nematode Caenorhabditis elegans: structure, regulation and biology. Prog. Mol. Subcell. Biol. 28, 61-78 (2002).

44. Abrahante, J. E., Miller, E. A. \& Rougvie, A. E. Identification of heterochronic mutants in Caenorhabditis elegans. Temporal misexpression of a collagen::green fluorescent protein fusion gene. Genetics 149, 1335-1351 (1998).

45. Mori, I. \& Ohshima, Y. Neural regulation of thermotaxis in Caenorhabditis elegans. Nature 376, 344-348 (1995).

46. Chen, Z. et al. Two insulin-like peptides antagonistically regulate aversive olfactory learning in C. elegans. Neuron 77, 572-585 (2013).

47. Harris, G. et al. Dissecting the serotonergic food signal stimulating sensorymediated aversive behavior in C. elegans. PLoS One 6, e21897 (2011).

48. Kuhara, A. \& Mori, I. Molecular physiology of the neural circuit for calcineurin-dependent associative learning in Caenorhabditis elegans. J. Neurosci. 26, 9355-9364 (2006).

49. Hendricks, M., Ha, H., Maffey, N. \& Zhang, Y. Compartmentalized calcium dynamics in a C. elegans interneuron encode head movement. Nature 487, 99-103 (2012).

50. Ibuka, N. \& Kawamura, H. Loss of circadian rhythm in sleep-wakefulness cycle in the rat by suprachiasmatic nucleus lesions. Brain Res. 96, 76-81 (1975).

51. Maquet, P. The role of sleep in learning and memory. Science 294, 1048-1052 (2001).

52. Ida, T. et al. Neuromedin $\mathrm{S}$ is a novel anorexigenic hormone. Endocrinology 146, 4217-4223 (2005).

53. Shousha, S. et al. Effect of neuromedin S on feeding regulation in the Japanese quail. Neurosci. Lett. 391, 87-90 (2006).

54. Tachibana, T., Matsuda, K., Khan, M. S., Ueda, H. \& Cline, M. A. Feeding and drinking response following central administration of neuromedin $\mathrm{S}$ in chicks. Comp. Biochem. Physiol. A. Mol. Integr. Physiol. 157, 63-67 (2010).

55. Atsuchi, K. et al. Centrally administered neuromedin S inhibits feeding behavior and gastroduodenal motility in mice. Horm. Metab. Res. 42, 535-538 (2010).

56. Maier, W., Adilov, B., Regenass, M. \& Alcedo, J. A neuromedin U receptor acts with the sensory system to modulate food type-dependent effects on C. elegans lifespan. PLoS Biol. 8, e1000376 (2010).

57. Moyle, W. R. et al. Co-evolution of ligand-receptor pairs. Nature 368, 251-255 (1994).

58. Schwarz, J., Lewandrowski, I. \& Bringmann, H. Reduced activity of a sensory neuron during a sleep-like state in Caenorhabditis elegans. Curr. Biol. 21, R983-R984 (2011).

59. Gallagher, T., Kim, J., Oldenbroek, M., Kerr, R. \& You, Y. J. ASI regulates satiety quiescence in C. elegans. J. Neurosci. 33, 9716-9724 (2013).

60. Davis, M. W. et al. Mutations in the Caenorhabditis elegans Na,K-ATPase alpha-subunit gene, eat-6, disrupt excitable cell function. J. Neurosci. 15, 8408-8418 (1995).

61. Nelson, M. D. \& Fitch, D. H. Overlap extension PCR: an efficient method for transgene construction. Methods Mol. Biol. 772, 459-470 (2011).
62. Stinchcomb, D. T., Shaw, J. E., Carr, S. H. \& Hirsh, D. Extrachromosomal DNA transformation of Caenorhabditis elegans. Mol. Cell Biol. 5, 3484-3496 (1985).

63. Mello, C. \& Fire, A. DNA transformation. Methods Cell Biol. 48, 451-482 (1995).

64. Thompson, O. et al. The Million Mutation Project: a new approach to genetics in Caenorhabditis elegans. Genome Res. 23, 1749-1762 (2013).

65. Belfer, S. et al. Caenorhabditis-in-drop array for monitoring C. elegans quiescent behavior. Sleep 36, 689-698G (2013).

66. Chao, M. Y., Komatsu, H., Fukuto, H. S., Dionne, H. M. \& Hart, A. C. Feeding status and serotonin rapidly and reversibly modulate a Caenorhabditis elegans chemosensory circuit. Proc. Natl Acad. Sci. USA 101, 15512-15517 (2004).

67. Zimmerman, J. E., Raizen, D. M., Maycock, M. H., Maislin, G. \& Pack, A. I. A video method to study Drosophila sleep. Sleep 31, 1587-1598 (2008).

68. Livak, K. J. \& Schmittgen, T. D. Analysis of relative gene expression data using real-time quantitative PCR and the 2(-Delta Delta C(T)) Method. Methods 25 402-408 (2001).

69. Hoogewijs, D., Houthoofd, K., Matthijssens, F., Vandesompele, J. \& Vanfleteren, J. R. Selection and validation of a set of reliable reference genes for quantitative sod gene expression analysis in C. elegans. BMC. Mol. Biol. 9, 9 (2008).

\section{Acknowledgements}

We thank Amita Sehgal, Liliane Schoofs and Lotte Frooninckx for comments. This work was supported by NIH grants T32HL07713 to M.D.N. (PI, Allan Pack), T31HL07953 to N.F.T. (PI, Allan Pack), R01NS064030 to D.M.R., a NARSAD Young Investigator Award to D.M.R., a McCabe Foundation grant to D.M.R., a Penn University Scholars Award to C.-C.Y. and by an Alfred P. Sloan Foundation Research Fellowship to C.F.-Y. Some strains were provided by the CGC, which is funded by NIH Office of Research Infrastructure Programs (P40 OD010440). The strain ARF240 was provided by A. Frand and the strain VM1345 was provided by A. Maricq.

\section{Author contributions}

M.D.N., N.F.T., C.F.-Y. and D.M.R. designed and performed experiments and wrote paper, J.B.G.-R. designed experiments, C.-C.Y. and C.J.S. performed experiments.

\section{Additional information}

Supplementary Information accompanies this paper at http://www.nature.com/ naturecommunications

Competing financial interests: The authors declare no competing financial interests.

Reprints and permission information is available online at http://npg.nature.com/ reprintsandpermissions/

How to cite this article: Nelson, M. D. et al. The neuropeptide NLP-22 regulates a sleeplike state in Caenorhabditis elegans. Nat. Commun. 4:2846 doi: 10.1038/ncomms3846 (2013). 